\section{Robust Region-of-Attraction Estimation}

Ufuk Topcu, Andrew K. Packard, Peter Seiler, and Gary J. Balas

\begin{abstract}
We propose a method to compute invariant subsets of the region-of-attraction for asymptotically stable equilibrium points of polynomial dynamical systems with bounded parametric uncertainty. Parameterindependent Lyapunov functions are used to characterize invariant subsets of the robust region-of-attraction. A branch-and-bound type refinement procedure reduces the conservatism. We demonstrate the method on an example from the literature and uncertain controlled short-period aircraft dynamics.
\end{abstract}

Index Terms-Branch-and-bound, parameter uncertainty, region-of-attraction (ROA).

\section{INTRODUCTION}

We consider the problem of computing invariant subsets of the region-of-attraction (ROA) for systems with polynomial vector fields and bounded parametric uncertainty. Since computing the exact ROA, even for systems with known dynamics, is hard, research has focused on determining Lyapunov functions whose sublevel sets characterize invariant subsets of the ROA [8], [9], [19]. Recent advances in polynomial optimization based on sum-of-squares (SOS) relaxations [12] are utilized to determine invariant subsets of the ROA for systems with known polynomial and/or rational dynamics solving optimization problems with matrix inequality constraints [7], [14], [15], [17], [21]. The literature on ROA analysis for systems with uncertain dynamics includes a generalization of Zubov's method [4] and an iterative algorithm that asymptotically gives the robust ROA for systems with time-varying perturbations [11]. Systems with parametric uncertainties are considered in [5], [13], [18]. The focus in [5] is on computing the largest sublevel set of a given Lyapunov function that can be certified to be an invariant subset of the ROA. References [5], [13] propose parameter-dependent Lyapunov functions which lead to potentially less conservative estimate of the ROA compared to parameter-independent Lyapunov functions at the expense of increased computational complexity.

This technical note follows [16], using bilinear sum-of-squares optimization to determine invariant subsets of the robust ROA. The differences lie in the allowed uncertain parameter dependence and the class of Lyapunov functions. The approach in [16] employs parameter-independent Lyapunov functions for systems whose vector field depends affinely on uncertain parameters known to lie in a given polytope, reminiscent of quadratic stability analysis [3], where a single quadratic Lyapunov function certifies the stability of an entire family of uncertain linear systems, usually described by a polytope of linear vector fields. Of course, using a common Lyapunov function tends to yield

Manuscript received July 14, 2008; revised February 01, 2009 and July 22, 2009. First published November 17, 2009; current version published January 13, 2010. This work was supported by the Air Force Office of Scientific Research, USAF, under Grant FA9550-05-1-0266. Recommended by Associate Editors S. Selikovsky and G. Chesi.

$\mathrm{U}$. Topcu is with the Control and Dynamical Systems, California Institute of Technology, Pasadena, CA 91125 USA (e-mail: utopcu@cds.caltech.edu).

A. K. Packard is with the Department of Mechanical Engineering, University of California, Berkeley, CA 94720 USA (e-mail: pack@me.berkeley.edu).

P. Seiler and G. J. Balas are with the Department of Aerospace Engineering and Mechanics, University of Minnesota, Minneapolis, MN 55455 USA (e-mail: peter.j.seiler@gmail.com, balas@aem.umn.edu).

Color versions of one or more of the figures in this technical note are available online at http://ieeexplore.ieee.org.

Digital Object Identifier 10.1109/TAC.2009.2033751 conservative results, and the restriction to polytopes of vector fields is undesirable. This technical note partially alleviates both of these limitations. First, vector fields are allowed to depend affinely on polynomial functions of the uncertain parameters, and we develop methods to cover these with a polytope of vector fields (so that [16] applies). Additionally, we propose a branch-and-bound type procedure [10] to partition the uncertainty set, computing a different parameter-independent Lyapunov function for each cell of the parameter space. Taken together, this collection implicitly defines a parameter-dependent Lyapunov function, $V(x, \delta)$ which is polynomial in $x$ (state) for fixed $\delta$ (uncertain parameter), and piecewise constant in $\delta$ for fixed $x$. We note that in robustness analysis involving time-invariant unknown parameters, it is common, [2], [22], to combine easily-computable sufficient conditions with branch-and-bound strategies, often yielding improved analysis results.

An alternate for the conservativeness of parameter-independent Lyapunov functions is using polynomially parameter-dependent Lyapunov functions as proposed in [5], [13]. Although SOS optimization can be used with parameter-dependent Lyapunov functions, the ensuing optimization problem is challenging because uncertain parameters are treated as additional independent variables in the SOS conditions, which can greatly affect the size of the semidefinite programs. Moreover, choosing a suitable and effective polynomially parameter-dependent basis for the Lyapunov function is not intuitive.

Finally, the methodology based on branch-and-bound, applied to robust ROA analysis here, is also applicable to local reachability and gain analysis of systems with parametric uncertainty.

Notation: $\mathbb{R}[x]$ is the set of polynomials in $x$ with real coefficients. For $\pi \in \mathbb{R}[x], \partial(\pi)$ denotes the degree of $\pi$. The subset $\Sigma[x]:=$ $\left\{\pi_{1}^{2}+\cdots+\pi_{m}^{2}: \pi_{1}, \ldots, \pi_{m} \in \mathbb{R}[x]\right\}$ is the set of SOS polynomials. For $\eta \in \mathcal{R}$ and $g: \mathcal{R}^{n} \rightarrow \mathcal{R}$, the $\eta$-sublevel set $\Omega_{g, \eta}$ of $g$ is defined as $\Omega_{g, \eta}:=\left\{x \in \mathcal{R}^{n}: g(x) \leq \eta\right\} . \mathcal{C}^{1}\left(\mathcal{R}^{n}\right)$ denotes the set of continuously differentiable, scalar valued functions on $\mathcal{R}^{n}$.

\section{ESTIMATION OF THE RobUST ROA OF SYSTEMS WITH PARAMETRIC UNCERTAINTY}

Consider the system governed by

$$
\dot{x}(t)=f(x(t), \delta)
$$

where $\delta \in \boldsymbol{\Delta} \subset \mathcal{R}^{m}$ is the vector of unknown parameters and $\boldsymbol{\Delta}$ is a known bounded polytope. For each $\delta \in \boldsymbol{\Delta}, f(\cdot, \delta): \mathcal{R}^{n} \rightarrow \mathcal{R}^{n}$ is locally Lipschitz and satisfies $f(0, \delta)=0$. The robust region-of-attraction (ROA) is the intersection of the ROAs for all systems governed by (1), i.e., $\bigcap_{\delta \in \Delta}\left\{\mathbf{x}_{0} \in \mathcal{R}^{n}: \lim _{t \rightarrow \infty} \varphi\left(t ; \mathbf{x}_{0}, \delta\right)=0\right\}$, where $\varphi\left(t ; \mathbf{x}_{0}, \delta\right)$ denotes the solution of (1) at time $t$ with initial condition $\mathbf{x}_{0}$ and fixed parameter value $\delta \in \Delta$. Trivial extensions of results found in classic texts [20] show that sublevel sets of appropriate Lyapunov functions are invariant subsets of the robust ROA. For any $D \subset \mathcal{R}^{m}$ and $V \in \mathcal{C}^{1}\left(\mathcal{R}^{n}\right)$, define $\mathcal{M}_{D, V}:=\bigcap_{\delta \in D}\left\{x \in \mathcal{R}^{n}: \nabla V(x) f(x, \delta)<0\right\}$.

Proposition 2.1: If there exist $\gamma>0$ and $V \in \mathcal{C}^{1}\left(\mathcal{R}^{n}\right)$ such that

$$
\begin{aligned}
& V(0)=0 \text { and } V(x)>0 \text { for all } x \neq 0, \\
& \Omega_{V, \gamma} \text { is bounded, and } \\
& \Omega_{V, \gamma} \backslash\{0\} \subset \mathcal{M}_{\Delta, V}
\end{aligned}
$$

hold, then for all $\mathbf{x}_{0} \in \Omega_{V, \gamma}$ and for all $\delta \in \boldsymbol{\Delta}, \varphi\left(t ; \mathbf{x}_{0}, \delta\right)$ exists, satisfies $\varphi\left(t ; \mathbf{x}_{0}, \delta\right) \in \Omega_{V, \gamma}$ for all $t \geq 0$, and $\lim _{t \rightarrow \infty} \varphi\left(t ; \mathbf{x}_{0}, \delta\right)=$ 0 , i.e., $\Omega_{V, \gamma}$ is an invariant subset of the robust ROA.

\section{$\triangleleft$}


Now restrict attention to a special case, where the dependence of $f$ on $\delta$ is affine, to obtain conditions equivalent to (4) but suitable for numerical verification (a generalization to polynomial dependence on $\boldsymbol{\delta}$ is treated in Section III). Assume that (1) is in the form

$$
\dot{x}(t)=f_{0}(x(t))+\sum_{i=1}^{m} \delta_{i} f_{i}(x(t))
$$

where $f_{0}, f_{1}, \ldots, f_{m}: \mathcal{R}^{n} \rightarrow \mathcal{R}^{n}$ are known locally Lipschitz functions and satisfy $f_{i}(0)=0$ for $i=0,1, \ldots, m$, and $\delta \in \Delta$. Further, denote the set of vertices (extreme points) of $\Delta$ by $\mathcal{E}_{\Delta}$. Then, the affine dependence of the vector field on $\delta$ trivially implies the following (e.g., [16]).

Proposition 2.2: For the vector field in (5) and $V \in \mathcal{C}^{1}\left(\mathcal{R}^{n}\right)$, $\mathcal{M}_{\boldsymbol{\Delta}, V}=\mathcal{M}_{\mathcal{E}_{\boldsymbol{\Delta}}, V}$.

Consequently, for any $V \in \mathcal{C}^{1}\left(\mathcal{R}^{n}\right)$ satisfying (2) and (3), and

$$
\Omega_{V, \gamma} \backslash\{0\} \subset \mathcal{M}_{\mathcal{E}_{\boldsymbol{\Delta}}, V}
$$

the sublevel set $\Omega_{V, \gamma}$ is an invariant subset of the robust ROA. In order to enlarge the computed invariant subset of the robust ROA by choice of $V$, we introduce a fixed, positive definite, convex function $p$, called the analysis shape factor and maximize $\beta$ while imposing the constraints (2)-(3), (6), and $\Omega_{p, \beta}:=\left\{x \in \mathcal{R}^{n}: p(x) \leq \beta\right\} \subseteq \Omega_{V, \gamma}$. This is written as an optimization problem

$$
\begin{gathered}
\beta_{\Delta}^{o p t}(\mathcal{V}):=\max _{V \in \mathcal{V}, \beta>0, \gamma>0} \beta \text { subject to } \\
(2),(3),(6), \text { and } \Omega_{p, \beta} \subseteq \Omega_{V, \gamma} .
\end{gathered}
$$

Here, $\mathcal{V} \subset \mathcal{C}^{1}\left(\mathcal{R}^{n}\right)$ denotes the set of candidate Lyapunov functions over which the maximum is computed. In practice, $p$ is problem-dependent and chosen by the analyst. Since the form of the certified inner estimate of the robust ROA is a sublevel set of $p$, the sublevel sets of $p$ should be well-understood (for in high-dimensions they cannot be visualized), and should reflect directionality/scaling information that the analyst is interested in learning with regard to the robust ROA. In order to relax the problem in (7) to a SOS programming problem, we require $f_{0}, f_{1}, \ldots, f_{m}$ and $p$ to be polynomials and restrict $V$ to be a polynomial in $x$ of fixed degree. Further, we use generalizations of the S-procedure [16] to obtain sufficient conditions for the set containment constraints in (7) and SOS conditions for polynomial nonnegativity [12]: if $\pi \in \Sigma[x]$, then $\pi$ is nonnegative.

Let $\mathcal{V}_{\text {poly }} \subseteq \mathcal{V}, \mathcal{S}_{1}, \mathcal{S}_{2}$, and $\mathcal{S}_{3}$ be prescribed finite-dimensional subsets of $\mathbb{R}[x]$, and denote $\mathcal{S}=\left(\mathcal{S}_{1}, \mathcal{S}_{2}, \mathcal{S}_{3}\right)$. For a polytopic subset $D$ of $\boldsymbol{\Delta}$ and positive definite polynomials $l_{1}$ and $l_{2}$ (typically $l_{i}(x)=$ $\epsilon_{i} x^{T} x$ with small scalars $\left.\epsilon_{i}\right)$, define $\beta_{D}\left(\mathcal{V}_{\text {poly }}, \mathcal{S}\right)$ as

$$
\begin{aligned}
& \beta_{D}\left(\mathcal{V}_{\text {poly }}, \mathcal{S}\right):=\max _{V \in \mathcal{V}_{\text {poly }}, \beta, \gamma, s_{1} \in \mathcal{S}_{1}, s_{2 \delta} \in \mathcal{S}_{2}, s_{3 \delta} \in \mathcal{S}_{3}} \beta \text { subject to } \\
& \quad s_{1} \in \Sigma[x], s_{2 \boldsymbol{\delta}} \in \Sigma[x], \quad s_{3 \delta} \in \Sigma[x], \text { for all } \delta \in \mathcal{E}_{D} \\
& \beta>0, \quad \gamma>0, \quad V(0)=0, \quad V-l_{1} \in \Sigma[x] \\
& -\left[(\beta-p) s_{1}+(V-\gamma)\right] \in \Sigma[x], \text { and } \\
& -\left[(\gamma-V) s_{2 \delta}+\nabla V\left(f_{0}+\sum_{i=1}^{m} \delta_{i} f_{i}\right) s_{3 \delta}+l_{2}\right] \in \Sigma[x]
\end{aligned}
$$$$
\text { for all } \delta \in \mathcal{E}_{D} \cdot(8 \mathrm{~d})
$$

The feasibility of the constraints in (8) is sufficient for the feasibility of the constraints in (7). Therefore, $\beta_{\Delta}\left(\mathcal{V}_{\text {poly }}, \mathcal{S}\right) \leq \beta_{\Delta}^{o p t}(\mathcal{V})$. Note that in (8a), since $\mathcal{E}_{D}$ is a finite set, $\left\{s_{2} \delta, s_{3 \delta}\right\} \delta \in \mathcal{E}_{D}$ is a finite set of polynomials in $x$, indexed by $\delta$.

The optimization in (8) is naturally converted to a bilinear semidefinite program (SDP), with 3 "types" of decision variables: the free parameters in $V$, the free parameters in the $s$ polynomials, and the free parameters introduced by the SOS constraints. The SDP is bilinear in the free parameters in $V$ and multipliers $s$, as evidenced by the product terms (e.g., $V s_{2}, \nabla V f s_{3 \delta}$, etc). We have made significant pragmatic progress in obtaining high-quality solutions to (8), using simulation to first derive a convex outer-bound on the set of feasible $V$ parameters [17], followed by coordinatewise optimization over $V$ and $\left(s_{1}, s_{2 \delta}, s_{3 \delta}\right)$. Nevertheless, the nonconvexity is not to be taken lightly, and any numerical attempt to compute $\beta_{D}\left(\mathcal{V}_{\text {poly }}, \mathcal{S}\right)$ must itself treated as a lower bound.

Finally, note that, if $l_{1}$ and $l_{2}$ have positive definite quadratic part, then the feasibility of (8) implies the robust stability of the uncertain linearized dynamics established with a common quadratic Lyapunov function. For systems with cubic vector fields, the feasibility of (8) is also necessary by the following theorem whose proof is in Appendix.

Theorem 2.1: Let $f_{0}, \ldots, f_{m}$ be cubic polynomials in $x$ satisfying $f_{0}(0)=\cdots=f_{m}(0)=0, P \succ 0, L_{1} \succ 0, L_{2} \succ 0, p(x)=x^{T} P x$, $l_{1}(x)=x^{T} L_{1} x$, and $l_{2}(x)=x^{T} L_{2} x$. For $\delta \in \Delta$, let $A_{\delta}$ be such that $A_{\delta} x$ is the linear (in $x$ ) part of $f_{0}(x)+\sum_{i=1}^{m} \delta_{i} f_{i}(x)$. If there exists $Q \succ 0$ satisfying $A_{\delta}^{T} Q+Q A_{\delta} \prec 0$ for all $\delta \in \mathcal{E}_{\boldsymbol{\Delta}}$, then the constraints in $(8)$ are feasible with $\partial(V)=\partial\left(s_{2 \delta}\right)=2$ and $\partial\left(s_{1}\right)=\partial\left(s_{3 \delta}\right)=0$. $\triangleleft$

\section{POLYNOMIAL PARAMETRIC UNCERTAINTY}

We extend Section II to systems with polynomial parametric uncertainty

$$
\dot{x}(t)=f_{0}(x(t))+\sum_{i=1}^{m} \boldsymbol{\delta}_{i} f_{i}(x(t))+\sum_{j=1}^{m_{p u}} g_{j}(\boldsymbol{\delta}) f_{m+j}(x(t))
$$

where $f_{0}, f_{1}, \ldots, f_{m}, f_{m+1} \ldots, f_{m+m_{p u}}: \mathcal{R}^{n} \rightarrow \mathcal{R}^{n}$ are vector valued polynomial functions satisfying $f_{0}(0)=\cdots=f_{m+m_{p u}}(0)=$ 0 , and $g_{1}, \ldots, g_{m_{p u}} \in \mathbb{R}[\boldsymbol{\delta}]$ are scalar valued polynomial functions, and $\delta$ takes values in a bounded polytope $\boldsymbol{\Delta}$. We begin with $m_{p u}=1$ (for simplicity) and then generalize for $m_{p u} \geq 1$.

Replacing $g_{1}(\boldsymbol{\delta})$ by an artificial parameter $\phi$, the dynamics in (9) can be written as

$$
\dot{x}(t)=f_{0}(x(t))+\sum_{i=1}^{m} \delta_{i} f_{i}(x(t))+\phi f_{m+1}(x(t)) .
$$

Our approach is based on covering the graph of $g$, $\left\{(\zeta, g(\zeta)) \in \mathcal{R}^{m+1}: \zeta \in \boldsymbol{\Delta}\right\}$, by a bounded polytope $\Gamma \subset \mathcal{R}^{m+1}$. Then, the dependence of the vector field in (10) on the parameters $(\boldsymbol{\delta}, \phi)$ is affine and $(\boldsymbol{\delta}, \phi)$ takes values in the bounded polytope $\Gamma$. Therefore, results from Section II are applicable for the system in (10) by replacing $\Delta$ by $\Gamma$.

A polytope $\Gamma$ covering the graph of $g$ can be obtained by bounding $g$ from above and below by affine functions $a_{u}^{T} \boldsymbol{\delta}+b_{u}$ and $a_{l}^{T} \boldsymbol{\delta}+b_{l}$ over the set $\boldsymbol{\Delta}$, namely $\Gamma\left(a_{l}, a_{u}, b_{l}, b_{u}\right):=$ $\left\{(\zeta, \psi) \in \mathcal{R}^{m+1}: \zeta \in \Delta, a_{l}^{T} \zeta+b_{l} \leq \psi \leq a_{u}^{T} \zeta+b_{u}\right\}$.

The volume of $\Gamma$ is a linear function of $a_{l}, a_{u}, b_{l}$, and $b_{u}$, $\operatorname{Volume}\left(\Gamma\left(a_{l}, a_{u}, b_{l}, b_{u}\right)\right)=\left(a_{u}-a_{l}\right)^{T} \int_{\Delta} \zeta d \zeta+\left(b_{u}-b_{l}\right) \int_{\Delta} d \zeta$. The polytope with smallest volume among such covering polytopes can be characterized via

$$
\begin{aligned}
\min _{a_{l}, a_{u}, b_{l}, b_{u}} & \text { Volume }\left(\Gamma\left(a_{l}, a_{u}, b_{l}, b_{u}\right)\right) \text { subject to } \\
& g(\boldsymbol{\delta})-\left(a_{l}^{T} \boldsymbol{\delta}+b_{l}\right) \geq 0 \text { and } \\
& g(\boldsymbol{\delta})-\left(a_{u}^{T} \boldsymbol{\delta}+b_{u}\right) \leq 0, \quad \forall \boldsymbol{\delta} \in \Delta .
\end{aligned}
$$

An upper bound for this minimal volume can be computed by a linear SOS optimization problem. To this end, let affine functions $h_{i}, i=1, \ldots, N$, provide an inequality description for $\Delta$, i.e., $\Delta=\left\{\zeta \in \mathcal{R}^{m}: h_{i}(\zeta) \geq 0, i=1, \ldots, N\right\}$. 
Proposition 3.1: The value of the optimization problem

$$
\min _{a, b, \sigma_{u i} \in \mathcal{S}_{u i}, \sigma_{l i} \in \mathcal{S}_{l i}} \operatorname{Volume}\left(\Gamma\left(a_{l}, a_{u}, b_{l}, b_{u}\right)\right)
$$

subject to $\sigma_{\alpha i} \in \Sigma[\delta], \quad \alpha \in\{l, u\}, \quad i=1, \ldots, N$

$$
\begin{aligned}
& -g(\boldsymbol{\delta})+\left(a_{u}^{T} \boldsymbol{\delta}+b_{u}\right)-\sum_{i=1}^{N} \sigma_{u i}(\boldsymbol{\delta}) h_{i}(\boldsymbol{\delta}) \in \Sigma[\boldsymbol{\delta}] \\
& g(\boldsymbol{\delta})-\left(a_{l}^{T} \boldsymbol{\delta}+b_{l}\right)-\sum_{i=1}^{N} \sigma_{l i}(\boldsymbol{\delta}) h_{i}(\boldsymbol{\delta}) \in \Sigma[\boldsymbol{\delta}]
\end{aligned}
$$

is an upper bound for (11). Here $\mathcal{S}$ 's are finite dimensional subsets of $\mathbb{R}[\boldsymbol{\delta}]$.

Proof: Since $\Delta$ is defined by $\left\{h_{i}(\delta) \geq 0\right\}_{i=1, \ldots, N}$, constraints in (12) imply that $a_{l}^{T} \delta+b_{l} \leq g(\delta) \leq a_{u}^{T} \delta+b_{u}$ for all $\delta \in \boldsymbol{\Delta}$.

Remark 3.1: Volume $\left.\left(\Gamma\left(a_{l}, a_{u}, b_{l}, b_{u}\right)\right)\right)=\operatorname{Volume}\left(\Gamma\left(0, a_{u}, 0, b_{u}\right)\right.$ -Volume $\left(\Gamma\left(0, a_{l}, 0, b_{l}\right)\right)$, hence the optimizing values of $a$ and $b$ in (12) can be computed by two smaller optimizations.

In case $m_{p u} \geq 1$, affine upper and lower bounds for $g_{1}, \ldots, g_{m_{p u}}$ (regardless of whether $g_{j}$ are polynomials) can be used to construct a polytope covering the graph of $\left(g_{1}, \ldots, g_{m_{p u}}\right)$ as formally stated in the following proposition from [1].

Proposition 3.2: For $j=1, \ldots, m_{p u}$, let $a_{l j}^{T} \delta+b_{l j}$ and $a_{u j}^{T} \delta+$ $b_{u j}$ be affine functions bounding $g_{j}$ over $\boldsymbol{\Delta}$ from below and above, respectively. Then, the polytope $\Gamma$ with the vertex set

$$
\begin{aligned}
\mathcal{E}_{\Gamma}:=\bigcup_{\zeta \in \mathcal{E}_{\boldsymbol{\Delta}}}\left\{\left(\zeta, \psi_{1}, \ldots, \psi_{m_{p u}}\right) \in \mathcal{R}^{m+m_{p u}}:\right. & \\
& \left.\psi_{j}=a_{\alpha j}^{T} \zeta+b_{\alpha j}, \alpha \in\{l, u\}, j=1, \ldots, m_{p u}\right\}
\end{aligned}
$$

contains the graph of $\left(g_{1}, \ldots, g_{m_{p u}}\right)$.

This gives one specific procedure to cover the graph of a vectorvalued multivariate polynomial by a convex polytope. Advances in graph covering strategies and quantifying the trade-off between the number of vertices and the volume of the covering polytope would be relevant to the robust ROA problem.

\section{BRANCH-AND-BOUND TYPE REFINEMENT IN THE PARAMETER SPACE}

The problem in (8), applied with $D=\boldsymbol{\Delta}$, computes invariant subsets of the robust ROA characterized by a single Lyapunov function though its results may be conservative: the certified invariant subset may be too small relative to the robust ROA. On the other hand, a less conservative estimate of the robust ROA is "obtained" by solving (8) for each $\delta \in \boldsymbol{\Delta}$ with $D=\{\delta\}$. For a subset $D \subseteq \boldsymbol{\Delta}$, define

$$
\beta_{D}^{*}\left(\mathcal{V}_{\text {poly }}, \mathcal{S}\right):=\min _{\delta \in D} \beta_{\{\delta\}}\left(\mathcal{V}_{\text {poly }}, \mathcal{S}\right) .
$$

Then, $\beta_{\Delta}\left(\mathcal{V}_{\text {poly }}, \mathcal{S}\right) \leq \beta_{\Delta}^{*}\left(\mathcal{V}_{\text {poly }}, \mathcal{S}\right)$. However, computing $\beta_{\Delta}^{*}\left(\mathcal{V}_{\text {poly }}, \mathcal{S}\right)$ requires solving an optimization problem for each $\delta \in \Delta$, and consequently is impractical. Next, we propose an informal "branch-and-bound" type procedure for computing lower and upper bounds for $\beta_{\Delta}^{*}\left(\mathcal{V}_{\text {poly }}, \mathcal{S}\right)$, i.e., localizing the value of $\beta_{\Delta}^{*}\left(\mathcal{V}_{\text {poly }}, \mathcal{S}\right)$. The method is based on computing a different Lyapunov function for each cell of a finite partition, $\mathcal{D}$, of $\boldsymbol{\Delta}$.

Branch-and-bound (B\&B) is an algorithmic method for global optimization based on two steps: the search region is partitioned into a union of smaller regions, or cells (branching) and then upper and lower bounds for the objective function restricted to each cell are computed (bounding) [10]. These steps are repeated, refining the partition each repetition (e.g., subdividing the cell with the worst lower bound). If the upper and lower bounds are such that their difference converges to zero uniformly as the size of the cell goes to zero, then the B\&B algorithm converges to a global optimum. Without such specific guarantees, steps are simply repeated until the gap between the upper and lower bounds gets suitably small or a maximum number of steps is reached. Additionally, for our problem, we take into account the polytopic covering described in Section III, and recompute this covering whenever any cell is subdivided.

The lower and upper bounds are defined over any polytope $D \subseteq \Delta$. Certainly $\beta_{D}\left(\mathcal{V}_{\text {poly }}, \mathcal{S}\right)$ is a lower bound for $\beta_{D}^{*}\left(\mathcal{V}_{\text {poly }}, \mathcal{S}\right)$. Upper bounds for $\beta_{D}^{*}\left(\mathcal{V}_{\text {poly }}, \mathcal{S}\right)$ can be obtained via divergent trajectories and infeasibility of certain necessary conditions for the constraints in (8). Let $\delta \in D$ and $\beta^{n c}(\delta)$ be the minimum value of $p$ attained on all nonconvergent trajectories of (5), with $\beta^{n c}(\delta):=\infty$ if there is no non-convergent trajectory. Since every trajectory entering an invariant subset of the robust ROA has to converge to the origin, $\Omega_{p, \beta^{n c}(\delta)}$ cannot be a subset of the robust ROA; hence, for any $\mathcal{V}_{\text {poly }}$ and $\mathcal{S}, \beta_{D}^{*}\left(\mathcal{V}_{\text {poly }}, \mathcal{S}\right)<$ $\beta^{n c}(\delta)$. Note, any non-convergent trajectory yields an upper bound on $\beta^{n c}(\delta)$, and consequently on $\beta_{D}^{*}\left(\mathcal{V}_{\text {poly }}, \mathcal{S}\right)$. In order to establish another upper bound, let $\beta>0$ and $\delta \in D$ be fixed. If there exists $V \in \mathcal{V}$ certifying that $\Omega_{p, \beta}$ is in the robust ROA through the constraints in (7), then $V$ has to be (i) positive for all nonzero $x \in \mathcal{R}^{n}$, (ii) less than or equal to 1 (without loss of generality) and decreasing along every trajectory of (5) (for this fixed $\delta$ ) starting in $\Omega_{p, \beta}$. Therefore, if no $V \in \mathcal{V}$ satisfies properties (i) and (ii), then there is no $V \in \mathcal{V}$ certifying that $\Omega_{p, \beta}$ is in the robust ROA via (7). The minimum such value, denoted $\beta^{l p}(\delta)$, is an upper bound on $\beta_{D}^{*}\left(\mathcal{V}_{\text {poly }}, \mathcal{S}\right)$. In the case $V$ is parameterized as $V(x)=\alpha^{T} z(x)$ with $z$ a vector of basis functions and $\alpha$ a vector of real scalar decision variables, constraints on $V$ along trajectories are affine constraints on $\alpha$; consequently, an upper bound on $\beta^{l p}(\delta)$ can be determined by simulation and linear programming (see [17]). As in all $B \& B$ algorithms, the minimum (over the subsets that partition $\Delta$ ) of these upper and lower bounds are upper and lower bounds for $\beta_{\Delta}^{*}\left(\mathcal{V}_{\text {poly }}, \mathcal{S}\right)$.

\section{IMPLEMENTATION ISSUES}

The optimization problem in (8) provides a recipe to compute invariant subsets of the robust ROA. However, the number of constraints in (8) and consequently the number of decision variables increase exponentially with $m+m_{p u}$ because (8d) contains a SOS constraint for each vertex value of the uncertainty polytope. The increase in the problem size may render (8) computationally challenging for even modest values of $m+m_{p u}$. An ad-hoc, sequential approach (from [16]), partially alleviates this difficulty, but without quantified, a priori guarantees as to its success. To this end, let $D$ be a polytopic subset of $\boldsymbol{\Delta}$ and $D_{\text {sample }}$ be a finite sample in $D$, with (typically) significantly fewer points than $\mathcal{E}_{D}$ :

- Solve (8) with $D_{\text {sample }}, \mathcal{V}_{\text {poly }}$, and $\mathcal{S}$ and call the optimizing Lyapunov function $V_{\text {sample }}$.

- For each $\delta \in \mathcal{E}_{D}$, compute

$$
\begin{aligned}
\gamma_{\delta}:= & \max _{0<\gamma, s_{2 \delta} \in \mathcal{S}_{2}, s_{3 \delta} \in \mathcal{S}_{3}} \gamma \text { subject to } \\
& s_{2 \delta} \in \Sigma[x], s_{3 \delta} \in \Sigma[x], \text { and } \\
& -\left[\left(\gamma-V_{\text {sample }}\right) s_{2 \delta}+\nabla V_{\text {sample }}\left(f_{0}+\left(\sum_{i=1}^{m} \delta_{i} f_{i}\right)\right)\right. \\
& \left.\times s_{3 \delta}+l_{2}\right] \in \Sigma[x]
\end{aligned}
$$

and define $\gamma^{\text {subopt }}:=\min \left\{\gamma_{\delta}: \delta \in \mathcal{E}_{D}\right\}$. At this point, $\Omega_{V_{\text {sample }, \gamma \text { subopt }} \text { is an invariant subset of the robust ROA. }}$ 
- Determine the largest sublevel set $\Omega_{p, \beta_{D}^{\text {subopt }}\left(\mathcal{V}_{\text {poly }}, \mathcal{S}\right)}$ of $p$ contained in $\Omega_{V_{\text {sample }}, \gamma^{\text {subopt }}}$ by solving

$$
\begin{aligned}
& \beta_{D}^{\text {subopt }}\left(\mathcal{V}_{\text {poly }}, \mathcal{S}\right):=\max _{s_{1} \in \mathcal{S}_{1}, \beta} \beta \\
& \text { subject to } s_{1} \in \Sigma[x] \\
& -\left[(\beta-p) s_{1}+V_{\text {sample }}-\gamma^{\text {subopt }}\right] \in \Sigma[x] .
\end{aligned}
$$

While this sequential procedure sacrifices optimality (i.e., for a given polytopic subset $D \subseteq \Delta, \beta_{D}^{\text {subopt }}\left(\mathcal{V}_{\text {poly }}, \mathcal{S}\right) \leq$ $\left.\beta_{D}\left(\mathcal{V}_{\text {poly }}, \mathcal{S}\right)\right)$, it has some practical advantages: For a fixed Lyapunov function candidate $V_{\text {sample }}$, constraints in (8d) (one SOS constraint for each vertex value of $D$ ) decouple. Therefore, it is possible to determine largest value of $\gamma$ such that $\Omega_{V_{\text {sample }}, \gamma} \subset\left\{x \in \mathcal{R}^{n}: \nabla V_{\text {sample }}(x) f(x, \delta)<0\right\}$ for every $\delta \in \mathcal{E}_{D}$ by solving (14) independently for each $\delta \in \mathcal{E}_{D}$.

Remark 5.1: If $D_{\text {sample }}$ is a singleton, the value $\beta_{D_{\text {sample }}}\left(\mathcal{V}_{\text {poly }}, \mathcal{S}\right)-\beta_{D}^{\text {subopt }}\left(\mathcal{V}_{\text {poly }}, \mathcal{S}\right)$ is always non-negative and can be interpreted as a measure of potential improvement in the lower bound for $\beta_{\Delta}^{*}\left(\mathcal{V}_{\text {poly }}, \mathcal{S}\right)$ by further subdivision of $D$. Therefore, it may be used as an informal stopping criterion in the $B \& B$ algorithm. However, we re-emphasize that $\beta_{D_{\text {sample }}}\left(\mathcal{V}_{\text {poly }}, \mathcal{S}\right)$ is computed solving a non-convex optimization problem, so that its use as an upper bound is ad hoc and referred to as a "quasi-upper" bound (e.g., see Fig. 2).

\section{EXAMPLES}

For the following examples, we implemented the sequential procedure from Section $\mathrm{V}$ using the method from [17] in the first step with $l_{1}(x)=l_{2}(x)=10^{-6} x^{T} x$ and $p(x)=x^{T} x$.

\section{A. An Example From the Literature}

Consider the system, [6], governed by

$$
\begin{aligned}
\dot{x}=\left[\begin{array}{c}
-x_{1} \\
3 x_{1}-2 x_{2}
\end{array}\right]+\left[\begin{array}{c}
-6 x_{2}+x_{2}^{2}+x_{1}^{3} \\
-10 x_{1}+6 x_{2}+x_{1} x_{2}
\end{array}\right] \delta & \\
& +\left[\begin{array}{c}
4 x_{2}-x_{2}^{2} \\
12 x_{1}-4 x_{2}
\end{array}\right] \delta^{2}
\end{aligned}
$$

with $\delta \in[0,1]=: \Delta$. We applied the refinement procedure with the initial partition $\{[0,1]\}$ for $\partial(V)=2$ and $\partial(V)=4$. Upper and lower bounds for $\beta_{\Delta}^{*}$ (top left for $\partial(V)=2$ and top right for $\left.\partial(V)=4\right)$ and certified invariant subsets of the robust ROA are shown in Fig. 1. In both cases, the first iteration (a parameter independent Lyapunov function for $\Delta,[16])$ and even a few more do not yield a certified region.

\section{B. Controlled Short-Period Aircraft Dynamics}

We apply the robust ROA analysis for uncertain controlled shortperiod aircraft dynamics (see Appendix for parameters)

$$
\begin{aligned}
\dot{x}_{p}=\left[\begin{array}{c}
c_{01}\left(x_{p}\right)+\delta_{1} c_{11}\left(x_{p}\right)+\delta_{1}^{2} q_{31}\left(x_{p}\right) \\
q_{02}\left(x_{p}\right)+\delta_{1} \ell_{12}^{T} x_{p}+\delta_{2} q_{22}\left(x_{p}\right)
\end{array}\right] \\
x_{1}
\end{aligned}
$$

where $x_{p}=\left[\begin{array}{lll}x_{1} & x_{2} & x_{3}\end{array}\right]^{T}, x_{1}, x_{2}$, and $x_{3}$ denote the pitch rate, the angle of attack, and the pitch angle, respectively, $c_{01}$ and $c_{11}$ are cubic polynomials, $q_{02}, q_{22}$, and $q_{31}$ are quadratic polynomials, $\ell_{12}$ and $\ell_{b}$
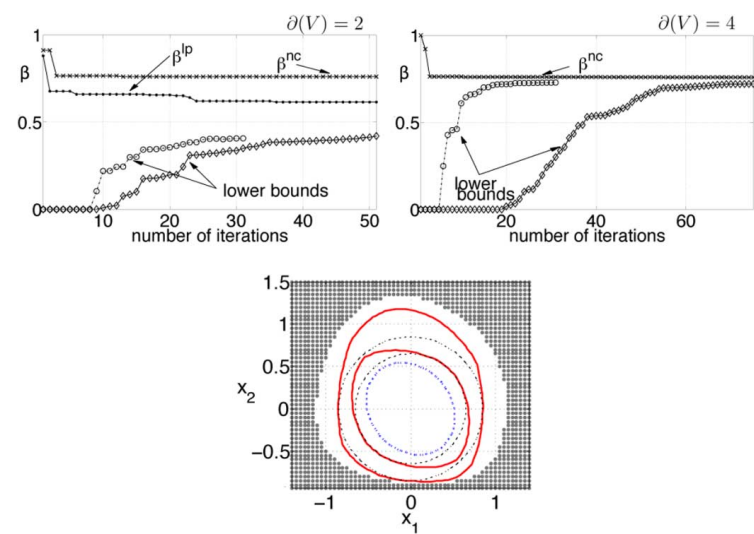

Fig. 1. Top figures: Bounds for $\beta_{[0,1]}^{*}$ versus number of $B \& B$ iterations with $\partial(V)=2$ (left) and $\partial(V)=4$ (right). Curves with "o" are for the lower bounds obtained by directly solving (8) with $D$ taken as the 4 vertices of the corresponding cell and curves with " $\diamond$ " are for the lower bounds obtained by applying the sequential procedure from Section $\mathrm{V}$ and taking $D_{\text {sample }}$ as the center of the corresponding cell. Bottom figure: Intersections of sublevel sets of $V$ 's certified to be in the robust ROA with $\partial(V)=2$ (inner red, solid curve) and $\partial(V)=4$ (outer red, solid curve), sublevel sets of $p$ certified to be in the robust ROA $\partial(V)=2$ (inner black, dashed curve) and $\partial(V)=4$ (outer black, dashed curve), estimate of the robust ROA reported in [6] (blue, dotted curve). Gray dots are the initial conditions of trajectories which do not converge to the origin for some $\delta \in[0,1]$.

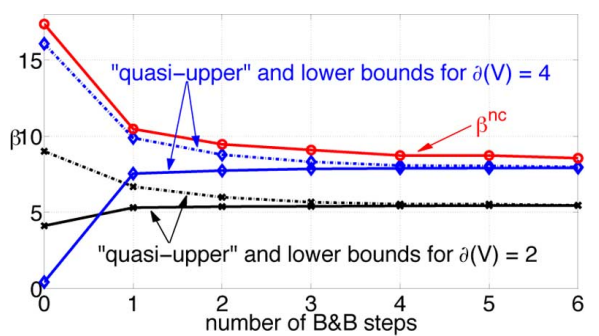

Fig. 2. Lower bounds for $\beta_{\Delta}^{*}$ with $\partial(V)=2$ (solid black with " $\times$ ") and $\partial(V)=4$ (solid blue curve with " $\nabla$ ") and $\beta^{n c}$ (solid red with "o") computed at the centers of the cells generated by the $B \& B$ Algorithm for the $\partial(V)=4$ run. Dashed curves are for (computed values of) $\beta_{\{\delta\}}$ where $\delta$ is the center of the cell with the smallest lower bound at the corresponding step of the $B \& B$ refinement procedure for $\partial(V)=2$ (dashed black with " $\times$ ") and $\partial(V)=4$ (dashed blue with " $\diamond$ ").

are vectors in $\mathcal{R}^{3}, b_{11}, b_{12}, b_{21}$, and $b_{22} \in \mathcal{R}$, and $u$, the elevator deflection, is the control input. $\delta_{1} \in[0.99,2.05]$ and $\delta_{2} \in[-0.1,0.1]$ model the variations in the center of gravity in the longitudinal direction and the mass, respectively. The control input is determined by $\dot{x}_{4}=-0.864 y_{1}-0.321 y_{2}$ and $u=2 x_{4}$, where $x_{4}$ is the controller state and the plant output $y=\left[\begin{array}{ll}x_{1} & x_{3}\end{array}\right]^{T}$. Define $x:=\left[\begin{array}{ll}x_{p}^{T} & x_{4}\end{array}\right]^{T}$. We applied the $\mathrm{B} \& \mathrm{~B}$ refinement procedure with $\partial(V)=2$ and $\partial(V)=4$ using the sequential implementation on 9 processors: after each $B \& B$ iteration, the cell with the smallest lower bound is subdivided into 3 subcells and cells with next three smallest lower bounds are sub-divided into 2 subcells. Fig. 2 shows the lower bounds and upper bounds. Smallest value of $p$ attained on divergent trajectories, $\beta^{n c}$, is 8.60 and obtained for $\left(\delta_{1}, \delta_{2}\right)=(2.039,-0.099)$ and the initial condition $(0.17,2.65,-0.10,1.24)$.

\section{Controlled Short-Period Aircraft Dynamics With First-Order Unmodeled Dynamics}

Consider the closed-loop system in Fig. 3 where uncertain first-order dynamics are introduced between the controller output $(v)$ and the plant input $(u)$ from Section VI-B

$$
\begin{aligned}
u(s) & =\left(1.25+G\left(s ; \delta_{3}, \delta_{4}\right)\right) v(s) \\
& =\left(1.25+0.75 \delta_{3} \frac{s-\delta_{4}}{s+\delta_{4}}\right) v(s) .
\end{aligned}
$$




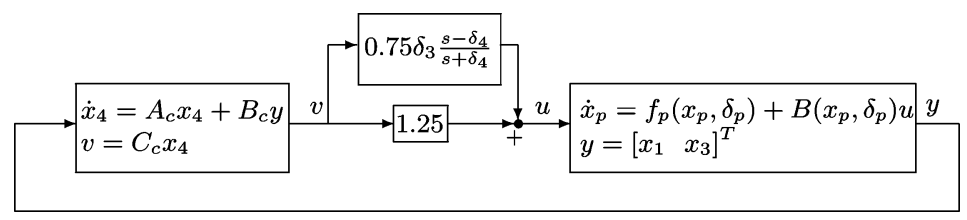

Fig. 3. Closed-loop system with the uncertain first-order dynamics between the controller and the plant $\left(\delta_{p}=\left(\delta_{1}, \delta_{2}\right)\right)$.

Here, $\delta_{3} \in[-1,1]$ and $\delta_{4} \in\left[10^{-2}, 10^{2}\right]$ are uncertain parameters and $G\left(s ; \delta_{3}, \delta_{4}\right)$ is introduced to examine the effect of unmodeled dynamics on the ROA. Let $\dot{x}_{5}=-\delta_{4} x_{5}-\delta_{4} v$ and $u=1.5 \delta_{3} x_{5}+$ $\left(1.25+0.75 \delta_{3}\right) v$ be a realization of $G$ and $x=\left[\begin{array}{lll}x_{p}^{T} & x_{4} & x_{5}\end{array}\right]^{T}$ denote the state of the closed loop dynamics. The resultant vector field is affine in $\delta_{1}, \delta_{2}, \delta_{3}, \delta_{4}, \delta_{1} \delta_{3}, \delta_{2} \delta_{3}$, and $\delta_{1}^{2}$, so the covering polytopes are in $\mathcal{R}^{7}$ with 128 vertices. We applied the sequential approach of Section V, using the center of each parameter hyper-rectangle as $D_{\text {sample }}$. The $B \& B$ algorithm partitioned the 4-dimensional $\delta$ space into 2221 regions, certifying $\Omega_{p, 2.8}$ in the robust ROA. Simply due to the large number of partitions, this indeed required significant time to solve. Nevertheless, every computational step consisted of either finding a Lyapunov function for a single, not-uncertain vector field; or assessing the certification power of a given Lyapunov function on a specified vector field. These individual computations are "simple," in that they involve no uncertainty, and decoupled. The complexity is the large number of computations performed, which taken together, yield a certified robust ROA for the uncertain vector field.

\section{CONCLUSION}

This technical note considers the problem of finding certified, innerestimates of the region-of-attraction for a certain class of uncertain nonlinear systems. At its core, the solution approach combines Lyapunov analysis, S-procedure relaxations, and SOS/SDP optimization. Four factors contribute to the problem complexity: number of state variables; degree of vector field; number of uncertain parameters; dependence of vector field on uncertain parameters. The challenges associated with state dimension and vector field degree (often large optimization problems) appear somewhat common across solution techniques. By contrast, the issues which arise from uncertainty are attacked using a variety of diverse techniques.

We address the difficulties due to parameter uncertainty through parallelization, partitioning the parameter space, solving a large number of (uncoupled) sub-problems. While the Lyapunov function for each sub-problem is independent of the uncertain parameter, the net result yields a parameter-dependent (piecewise-constant in the parameter) Lyapunov function. This is an alternative to more direct approaches which use explicitly parameter-dependent Lyapunov functions, e.g., [5], [13], and a single optimization (with additional indeterminate and decision variables, used to represent the uncertain parameters and capture their constraints) to solve the problem.

Of course, the question of how fine the parameter space partition must be before the proposed method yields a certified robust ROA is still largely open, so it is impossible to say that one approach is superior/inferior to another. Similarly, we do not claim that the proposed strategy is practical for all instances of systems modeled by (9). Indeed, large numbers of uncertain parameters, entering the dynamics in complex ways might require an untenable level of parameter space partitioning to yield a positive result. Nevertheless, we have illustrated the approach on several academic, but nontrivial, examples, including a 5-state, 4-parameter model with non-affine parameter dependence. Moreover, for cubic (in state) vector fields, we have a (weak) positive result which follows from Theorem 2.1, namely for any specific partition of the parameter space, if over each cell, the linearized uncertain dynamics are quadratically stable, then the certification conditions (8) are guaranteed to be feasible (with analytically derived choices for the decision variables). Among other things, this implies that the uncertain linearization could provide insight into the level of parameter space division needed for robust region-of-attraction certification.

\section{APPENDIX}

Let $z(x)$ be a vector of all monomials of degree 2 with no repetition and $n_{z}$ be its length.

Lemma 9.1: Let $Q \in \mathcal{R}^{n}$ and $Q=Q^{T} \succeq 0$. Then there exists a positive definite matrix $H \in \mathcal{R}^{n_{z} \times n_{z}}$ such that $x^{T} x x^{T} Q x=$ $z(x)^{T} H z(x)$.

Proof: Let $L_{i} \in \mathcal{R}^{n \times n_{z}}$ be such that $L_{i} z(x)=x_{i} x$, then $\left(x^{T} x\right) x^{T} Q x=\sum_{i=1}^{n}\left(x_{i} x\right)^{T} Q\left(x_{i} x\right)=\sum_{i=1}^{n} z(x) L_{i}^{T} Q L_{i} z(x)=$ $z(x)^{T} H z(x)$. Note that $L=\left[L_{1}^{T} \ldots L_{n_{z}}^{T}\right]^{\bar{T}}$ has full column rank since every entry of $z(x)$ is $x_{j} x_{k}$ for some $1 \leq j, k \leq n$; consequently, $H=L^{T}\left(I_{n} \otimes Q\right) L \succ 0$.

Proof of Theorem 2.1: Let $\tilde{Q} \succ 0$ satisfy $A_{\delta} \tilde{Q}+\tilde{Q} A_{\delta} \preceq-2 L_{2}$, for all $\delta \in \mathcal{E}_{\Delta}$, and $\tilde{Q} \succeq L_{1}$ (by scaling $Q$ ). Let $\epsilon:=\lambda_{\min }\left(L_{2}\right)$, $V(x):=x^{T} \tilde{Q} x$, and $H \succ 0$ be a Gram matrix for $\left(x^{T} x\right) V(x)$ (by Lemma 9.1). Let $M_{2 \delta} \in \mathcal{R}^{n \times n_{z}}$ and $M_{3 \delta}=M_{3 \delta}^{T} \in \mathcal{R}^{n_{z} \times n_{z}}$ be such that $x^{T} M_{2 \delta} z(x)$ and $z(x)^{T} M_{3 \delta} z(x)$ are cubic and quartic (in $x$ ) parts of $\nabla V\left(f_{0}(x)+\sum_{i=1}^{m} \delta_{i} f_{i}(x)\right)$, respectively. Define $s_{1}(x):=\lambda_{\max }(\tilde{Q}) / \lambda_{\min }(P), s_{2 \delta}(x):=\alpha_{2 \delta} x^{T} x$ with $\alpha_{2 \delta}:=\lambda_{\max }\left(M_{3 \delta}^{+}+(1 / 2 \epsilon) M_{2 \delta}^{T} M_{2 \delta}\right) / \lambda_{\min }(H)$ (where for a symmetric matrix $\Lambda, \Lambda^{+}$denotes the projection on the positive semidefinite cone), $s_{3 \delta}(x):=1, \gamma:=\min \left\{\epsilon /\left(2 \alpha_{2 \delta}\right): \delta \in \mathcal{E}_{\Delta}\right\}$, and $\beta:=\gamma /\left(2 s_{1}\right)$. Then, $V-l_{1}$ and $-\left[(\beta-p) s_{1}+(V-\gamma)\right]$ are SOS since they are positive semidefinite quadratic polynomials. For $\delta \in \mathcal{E}_{\boldsymbol{\Delta}}, b_{\delta}(x):=-\left[(\gamma-V) s_{2 \delta}+(\partial V / \partial x) f_{\delta} s_{3 \delta}+l_{2}\right]=$ $\left[\begin{array}{ll}x^{T} & z(x)^{T}\end{array}\right] B_{\delta}\left[x^{T} z(x)^{T}\right]^{T}$, where

$$
\begin{aligned}
B_{\delta} & =\left[\begin{array}{cc}
-\gamma \alpha_{2 \delta} I-L_{2}-\left(A_{\delta}^{T} \tilde{Q}+\tilde{Q} A_{\delta}\right) & \frac{-M_{2 \delta}}{2} \\
\frac{-M_{2 \delta}^{T}}{2} & \alpha_{2 \delta} H-M_{3 \delta}
\end{array}\right] \\
& \succeq\left[\begin{array}{cc}
\frac{\epsilon}{2} I & \frac{-M_{2 \delta}}{2} \\
\frac{-M_{2 \delta}^{T}}{2} & \alpha_{2 \delta} H-M_{3 \delta}
\end{array}\right] .
\end{aligned}
$$

Note that $\alpha_{2 \delta} H=\left(\lambda_{\max }\left(M_{3 \delta}^{+}+(1 / 2 \epsilon) M_{2 \delta}^{T} M_{2 \delta}\right) / \lambda_{\min }(H)\right) H$ $\succeq \lambda_{\max }\left(M_{3 \delta}^{+}+(1 / 2 \epsilon) M_{2 \delta}^{T} M_{2 \delta}\right) I \succeq \lambda_{\max }\left(M_{3 \delta}+(1 / 2 \epsilon) M_{2 \delta}^{T}\right.$ $\left.M_{2 \delta}\right) I \succeq M_{3 \delta}+(1 / 2 \epsilon) M_{2 \delta}^{T} M_{2 \delta}$. Consequently, $B_{\delta}$ is positive semidefinite by the Schur complement formula applied to the far left term in (17) and $b_{\delta} \in \Sigma[x]$.

Parameters for the uncertain controlled short-period aircraft dynamics: $c_{01}\left(x_{p}\right)=-0.24366 x_{2}^{3}+0.082272 x_{1} x_{2}+$ $0.30492 x_{2}^{2}+0.015426 x_{2} x_{3}-3.1883 x_{1}-2.7258 x_{2}-0.59781 x_{3}$ $\ell_{b}=\left[\begin{array}{lll}0 & -0.041136 & 0\end{array}\right]^{T} ; b_{11}=1.594150 ; q_{02}\left(x_{p}\right)=$ $-0.054444 x_{2}^{2}+0.10889 x_{2} x_{3}-0.054444 x_{3}^{2}+0.91136 x_{1}-$ $0.64516 x_{2}-0.016621 x_{3} ; b_{21}=0.0443215 ; c_{11}\left(x_{p}\right)=$ $0.30765 x_{2}^{3}+0.099232 x_{2}^{2}+0.12404 x_{1}+0.90912 x_{2}+0.023258 x_{3} ;$ $b_{12}=-0.06202 ; \ell_{12}=\left[\begin{array}{lll}0 & 0.00045754 & 0\end{array}\right]^{T} ; q_{22}\left(x_{p}\right)=$ $-0.054444 x_{2}^{2}+0.10889 x_{2} x_{3}-0.054444 x_{3}^{2}-0.6445 x_{2}-$ $0.016621 x_{3} ; b_{22}=0.044321$. 


\section{REFERENCES}

[1] F. Amato, F. Garofalo, and L. Glielmo, "Polytopic coverings and robust stability analysis via Lyapunov quadratic forms," in Variable Structure and Lyapunov Control. : : Springer-Verlag, 1994, pp. 269-288.

[2] V. Balakrishnan, S. Boyd, and S. Balemi, "Branch and bound algorithm for computing the minimum stability degree of parameter-dependent linear systems," Int. J. of Robust and Nonlinear Control, vol. 1, no. 4, pp. 295-317, 1991.

[3] B. R. Barmish, "Necessary and sufficient conditions for quadratic stabilizability of an uncertain system," Journal of Optimization Theory and Applications, vol. 46, no. 4, pp. 399-408, 1985.

[4] F. Camilli, L. Grune, and F. Wirth, "A generalization of Zubov's method to perturbed systems," SIAM Journal on Control and Optimization, vol. 40, no. 2, pp. 496-515, 2001.

[5] G. Chesi, "Estimating the domain of attraction of uncertain poynomial systems," Automatica, vol. 40, pp. 1981-1986, 2004.

[6] G. Chesi, "On the estimation of the domain of attraction for uncertain polynomial systems via lmis," in Proc. Conf. on Decision and Control, Bahamas, 2004, pp. 881-886.

[7] G. Chesi, A. Garulli, A. Tesi, and A. Vicino, "LMI-based computation of optimal quadratic Lyapunov functions for odd polynomial systems," Int. J. Robust Nonlinear Control, vol. 15, pp. 35-49, 2005.

[8] E. J. Davison and E. M. Kurak, "A computational method for determining quadratic Lyapunov functions for nonlinear systems," Automatica, vol. 7, pp. 627-636, 1971.

[9] R. Genesio, M. Tartaglia, and A. Vicino, "On the estimation of asymptotic stability regions: State of the art and new proposals," IEEE Transaction on Automatic Control, vol. 30, no. 8, pp. 747-755, 1985.

[10] E. L. Lawler and D. E. Wood, "Branch-and-bound methods: A survey," Operations Research, vol. 14, no. 4, pp. 679-719, 1966.

[11] A. Paice and F. Wirth, "Robustness analysis of domains of attraction of nonlinear systems," in Proceedings of the Mathematical Theory of Networks and Systems, 1998, pp. 353-356.

[12] P. Parrilo, "Semidefinite programming relaxations for semialgebraic problems," Mathematical Programming Series B, vol. 96, no. 2, pp. 293-320, 2003.

[13] W. Tan, "Nonlinear Control Analysis and Synthesis Using Sum-ofSquares Programming," Ph.D., Berkeley, UC, 2006.

[14] W. Tan and A. Packard, "Stability region analysis using polynomial and composite polynomial Lyapunov functions and sum-of-squares programming," IEEE Transactions on Automatic Control, vol. 53, pp. $565-571,2008$.

[15] B. Tibken and Y. Fan, "Computing the domain of attraction for polynomial systems via BMI optimization methods," in Proc. American Control Conf., Minneapolis, MN, 2006, pp. 117-122.

[16] U. Topcu and A. Packard, "Local stability analysis for uncertain nonlinear systems," IEEE Transactions on Automatic Control, 2008.

[17] U. Topcu, A. Packard, and P. Seiler, "Local stability analysis using simulations and sum-of-squares programming," Automatica, vol. 44, pp. 2669-2675, 2008.

[18] A. Trofino, "Robust stability and domain of attraction of uncertain nonlinear systems," in Proc. American Control Conf., Chicago, IL, 2000, pp. 3707-3711.

[19] A. Vannelli and M. Vidyasagar, "Maximal Lyapunov functions and domains of attraction for autonomous nonlinear systems," Automatica, vol. 21, no. 1, pp. 69-80, 1985.

[20] M. Vidyasagar, Nonlinear Systems Analysis, 2nd ed. Englewood Cliffs, NJ: Prentice Hall, 1993.

[21] T.-C. Wang, S. Lall, and M. West, "Polynomial level-set methods for nonlinear dynamical systems analysis," in Proc. Allerton Conf. Commun., Control, Comput., Allerton, IL, 2005 [Online]. Available: http://cslgreenhouse.csl.illinois.edu/allerton/archives/allerton05/ PDFs/Papers/II_C_6.pdf

[22] P. M. Young, M. P. Newlin, and J. C. Doyle, " $\mu$ analysis with real parametric uncertainty," in Proc. Conf. Decision Control, 1991, pp. $1251-1256$.

\section{A Switching Anti-windup Design Using Multiple Lyapunov Functions}

Liang Lu and Zongli Lin, Fellow, IEEE

\begin{abstract}
This technical note proposes a switching anti-windup design, which aims to enlarge the domain of attraction of the closed-loop system. Multiple anti-windup gains along with an index function that orchestrates the switching among these anti-windup gains are designed based on the min function of multiple quadratic Lyapunov functions. In comparison with the design of a single anti-windup gain which maximizes a contractively invariant level set of a single quadratic Lyapunov function as a way to enlarge the domain of attraction, the use of multiple Lyapunov functions and switching in the proposed design allows the union of the level sets of the multiple Lyapunov functions, each of which is not necessarily contractively invariant, to be contractively invariant and within the domain of attraction. As a result, the resulting domain of attraction is expected to be significantly larger than the one resulting from a single anti-windup gain and a single Lyapunov function. Indeed, simulation results demonstrate such a significant improvement.
\end{abstract}

Index Terms-Actuator saturation, anti-windup, composite Lyapunov functions, domain of attraction, switching systems.

\section{INTRODUCTION}

Anti-windup is a traditional approach to dealing with actuator saturation. The idea is to augment the closed-loop system that was designed without taking actuator saturation into consideration so that the negative effect of actuator saturation is weakened. Earlier works on anti-windup design try to minimize the effect of saturation in a direct way by reducing the difference between the input and output of the actuators (see, for example, [1], [8]).

Later works on anti-windup aim to reduce the effect of saturation indirectly by trying to improve the closed-loop stability and performances (see, for example, [3], [14], [16]-[18], [27] and the references therein). One of the earliest works that address the stability of control systems with anti-windup compensation involves the application of the scalar Popov and circle criteria ([9]). Stability analysis of multivariable systems with anti-windup compensation was carried out in ([15], [22]). The $\mathcal{L}_{2}$ formalism was introduced in [24] and adopted by several other authors (see, for example, [11], [25]).

More recently, an anti-windup design algorithm was developed with the explicit goal of enlarging the domain of attraction of the resulting system [3]. By expressing a saturated linear feedback law on the convex hull of some auxiliary linear feedbacks and using a single quadratic Lyapunov function, a set of conditions under which an ellipsoid, as a level set of the Lyapunov function, is contractively invariant and thus inside the domain of attraction, are established in terms of bilinear matrix inequalities in the auxiliary feedback gains, the positive definite matrix that defines the quadratic Lyapunov function and the anti-windup compensation gain. The design of the anti-windup gain

Manuscript received October 18, 2008; revised February 28, 2009. First published November 17, 2009; current version published January 13, 2010. This work was supported in part by NASA Langley Research Center under the contract NNX08AC80A. Recommended by Associate Editor F. Wu.

L. Lu is with the Department of Automation, Shanghai Jiao Tong University, Shanghai, China (e-mail: liangup@gmail.com).

Z. Lin is with the Charles L. Brown Department of Electrical and Computer Engineering, University of Virginia, Charlottesville, VA 22904-4743 USA (e-mail: zl5y@virginia.edu).

Color versions of one or more of the figures in this technical note are available online at http://ieeexplore.iee.org.

Digital Object Identifier 10.1109/TAC.2009.2033753 